Z Rheumatol 2013 $\cdot 72: 947$

DOI 10.1007/s00393-013-1192-z

Online publiziert: 4. Dezember 2013

(c) Springer-Verlag Berlin Heidelberg 2013

\title{
O. Distler
}

Klinik für Rheumatologie, UniversitätsSpital Zürich

\section{Frühkollagenosen}

Während die sog. Früharthritis heutzutage einen festen Bestandteil der klinischen Praxis von Rheumatologen darstellt, ist der Begriff der Frühkollagenosen weit weniger gängig. Dabei gelten die Beweggründe, die zur Etablierung von Früharthritissprechstunden und besonderen Programmen zur Früherkennung spezifischer Arthritiden geführt haben, grundsätzlich auch für Kollagenosen. Beispielsweise wäre eine Früherkennung von schwer verlaufenden Kollagenosen anhand einfacher Vorhersagealgorithmen von großem klinischem Nutzen. Optimal wären Instrumente, die eine Kollagenose bereits im präklinischen Stadium erkennen können, bevor ein Organschaden aufgetreten ist.

In diesem Zusammenhang stellen sich aber eine Reihe wichtiger Fragen:

- Ist eine präklinische Erkennung für Multisystemerkrankungen wie die Kollagenosen mit zumutbarem Aufwand überhaupt möglich?

- Wie gut sind die Vorhersagealgorithmen validiert - angesichts der Tatsache, dass es sich um seltene Erkrankungen handelt?

- Anders als bei der rheumatoiden Arthritis stehen für viele Kollagenosen weit weniger gut etablierte Therapien zur Verfügung. Hätte eine solche Früherkennung dann überhaupt (therapeutische) Konsequenzen?

- Können durch therapeutische Frühinterventionen und/oder intensivierte Screeningverfahren spätere Organschäden verhindert werden und wie gut ist dies durch Studien belegt?

- Wie hoch ist das Kosten-Nutzen-Risiko derartiger Therapien im Hinblick auf relevante Nebenwirkungen?

Dieses Spannungsfeld zwischen einem sicherlich vorhandenen Bedarf und großen methodischen Herausforderungen soll im aktuellen Themenschwerpunktheft Frühkollagenosen näher beleuchtet werden.
Ein Fokus ist dabei auf praktisch relevante Erkenntnisse zum frühen Verlauf bei Kollagenosen gelegt worden. R. Fischer-Betz und M. Schneider besprechen in ihrem Beitrag das breite Krankheitsspektrum des systemischen Lupus erythematodes, der gerade in Frühstadien große Herausforderungen an die Differenzialdiagnose stellt. Durch optimierte Analysen von Registern und durch weitere Initiativen ist es in den letzten Jahren bei den Frühformen der systemischen Sklerose zu einem besonders schnellen Erkenntniszuwachs gekommen, auf den M. Frerix, F. Meier und U. Müller-Ladner in zwei Artikeln eingehen. B. Maurer behandelt in ihrer Arbeit klinische und diagnostische Merkmale der Frühformen von idiopathischen inflammatorischen Myopathien, deren Spektrum sich in jüngerer Vergangenheit deutlich erweitert hat.

Viel Freude bei der Lektüre wünscht Ihnen

Ihr
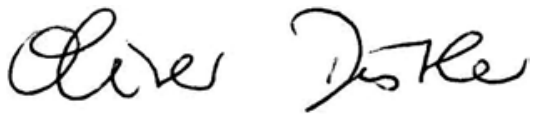

Prof. Dr. O. Distler

\section{Korrespondenzadresse}

Prof. Dr. O. Distler

Klinik für Rheumatologie,

UniversitätsSpital Zürich

Gloriastr. 25, 8091 Zürich

Schweiz

oliver.distler@usz.ch

Interessenkonflikt. O. Distler weist auf folgende Beziehungen hin: Prof. Dr. O. Distler hat/hatte eine beratende Funktion inne und/oder hat Forschungsförderung erhalten von Actelion, Bayer, Roche, GSK, Biogen, Pfizer, Ergonex, BMS, Sanofi-Aventis, Sinoxa, United BioSource Corporation, medac, Biovitrium, Boehringer Ingelheim Pharma, Novartis, 4 D Science und Active Biotec im Bereich der potenziellen Behandlung von Bindegewebserkrankungen und ihren Komplikationen. 